\title{
Clinical potential of oligonucleotide therapeutics against pancreatic cancer
}

Kazuki Takakura $^{1, *}$, Atsushi Kawamura ${ }^{1}$, Yuichi Torisu ${ }^{1}$, Shigeo Koido ${ }^{1}$, Naohisa Yahagi ${ }^{2}$, Masayuki Saruta ${ }^{1}$

${ }^{1}$ Division of Gastroenterology and Hepatology, Department of Internal Medicine, The Jikei University School of Medicine, Tokyo, Japan.

${ }^{2}$ Division of Research and Development for Minimally Invasive Treatment, Cancer Center, Keio University School of Medicine, Tokyo, Japan.

*Correspondence to: ktakakura@jikei.ac.jp; Tel.: +81-3-3433-1111 


\begin{abstract}
:
Although there is a several array of diagnostic and therapeutic choices for pancreatic cancer in recent years, a crucial medical approach for the refractory disease is still needed. Oligonucleotide therapeutics, such as those based on antisense RNAs, RNA interference, aptamers and decoys, are promising agents against pancreatic cancer because they identify a specific nucleotide sequence or protein and interfere with gene expression as molecular-targeted agents. Within just the past quarter-century, the diversity and feasibility of these drugs as diagnostic or therapeutic tools have dramatically increased. Actually, there have been several clinical and preclinical studies of oligonucleotides for patients with pancreatic cancer so far. To support the discovery of effective diagnostic or therapeutic options by using oligonucleotide-based strategies in the absence of satisfactory therapies for long-term survival and the rising trend of diseases, we summarize the current clinical trials of oligonucleotide therapeutics for pancreatic cancer patients with underlying preclinical or scientific data and focus on the possibility of oligonucleotides to target pancreatic cancer in clinical implications.
\end{abstract}

Keywords: Oligonucleotide therapeutics; RNA interference; antisense; aptamer; decoy; pancreatic cancer 


\section{Introduction}

Oligonucleotide therapeutics is a general term for state-of-the-art molecular-targeted agents that have chain-like structures of several nucleic acids, such as deoxyribonucleic acid (DNA) and ribonucleic acid (RNA), and they inhibit gene expression or impede protein function by binding a specific nucleotide sequence or protein [1]. In contrast to gene-based treatments that induce gene expression from a specific DNA sequence and yield a protein with some biofunction, a therapeutic nucleic acid, which is a chemosynthetic product, acts directly in a living body without going through gene expression in oligonucleotides. Therefore, oligonucleotide therapeutics have a high specificity and can target molecules that cannot be controlled by conventional drugs, such as mRNA or noncoding RNA, resulting in the development of innovative drugs against cancers and genetic diseases. Representative oligonucleotide therapeutics include antisense oligonucleotides (ASOs) and RNA interference (RNAi), especially small interfering RNA (siRNA), microRNAs (miRNAs), aptamers and decoys, and among them, ASO and RNAi have been further developed compared with the others so far.

The latest review articles show that pancreatic cancer is recognized as a malignant tumor with poor prognosis based on the delayed diagnosis and the high refractoriness to any available therapeutic approaches $[2,3]$. Thus, pancreatic cancer is receiving extensive attention in research on pharmaceutical agents in the absence of satisfactory treatments for long-term survival and the rising trend of diseases. Under such a situation, the clinical applications of oligonucleotide therapeutics for patients with pancreatic cancer have been in progress. Indeed, there have been a total of 9 clinical trials of oligonucleotide therapeutics that have been conducted for patients with pancreatic cancer that received National Institutes of Health (NIH) approval, while no trials of aptamers or decoys were conducted. A clinical trial of miRNA-25 in the diagnosis of pancreatic cancer from China was approved by NIH. Moreover, a promising clinical trial of carbohydrate sulfotransferase 15 (CHST15)-siRNA (STNM01) for patients with pancreatic cancer has been completed in Japan. In our previous study, CHST15, a specific enzyme that biosynthesizes chondroitin sulfate-E (CS-E), which is highly expressed in human pancreatic cancer cells, was a key player in tumor growth, and the effect of STNM01 on tumor cell proliferation in vitro and growth in vivo was demonstrated [4].

Here, we summarize current clinical trials of oligonucleotide therapeutics for patients with pancreatic cancer (Table 1), along with preclinical or scientific evidence of drug efficacy, focusing on the possibility of oligonucleotides as a potential diagnostic or therapeutic target for pancreatic cancer. 


\section{Effects of oligonucleotide therapeutics on disease progression}

The concept of ASOs started with a report from Belikova and colleagues on the first oligonucleotides in 1967 [5], and later reports from Zamecnik et al. definitively established the concept $[6,7]$. Moreover, a crucial study from Matsukura et al. promoted the large expansion of ASOs for various clinical applications [8]. Aptamers were first mentioned as a different kind of oligonucleotide in research about the regulation of human immunodeficiency virus-1 (HIV-1) in 1989 [9]. Thereafter, Fire and colleagues discovered RNAi as a breakthrough application of oligonucleotides in Caenorhabditis elegans in 1998 [10]. Since then, RNAi has become a major tool for various types of laboratory study, accounting for the first application of an RNAi strategy was successfully assessed by using a siRNA directed against the mRNA encoding the Nprotein of respiratory syncytial virus (RSV) [11]. In addition, miRNAs were found as an available option for oligonucleotides in 1993 [12], and it is assumed that the first clinical trial related to a decoy strategy was performed for HIV-1 infection, as with aptamers [13].

Taken together, studies from only the past 20 years have yielded diverse advancements in oligonucleotide research, and the potential targets for both diagnostic and therapeutic purposes might be unlimited based on the different action mechanisms. Although the mechanism by which oligonucleotides have their effects mostly depends on their structure and chemistry, the major premise of controlling the expression of targeted gene products is immutable. Due to the different mechanisms of each oligonucleotide, they act as inhibitors at different phases in disease progression. As shown in Figure 1, decoys work against transcription factors of targeted DNA at the earliest phase; subsequently, antisense RNA, siRNA and miRNA act on targeted RNAs; and finally, aptamers directly inhibit proteins involved in disease development. Their common point is that all of them act as inhibitors of disease progression by interfering with gene expression.

On the basis of diverse preclinical research progress, the current clinical trials of oligonucleotide therapeutics for patients with pancreatic cancer listed on the US website (http://Clinicaltrials.gov.) are summarized in Table 1.

\section{ASO}

When the antisense strategy was first introduced, it was recognized that ASOs could represent a specific, systemic gene silencing strategy. Successful development of such a strategy could allow an almost endless variety of human diseases to be treated, provided that a particular gene had been identified and characterized for the disease. There have 
already been several advanced ASO and RNAi agents to reach clinical trials; thus, we focus mainly on the clinical trials in this chapter.

ISIS-2503, a 20-mer ASO that hybridizes to the 5'-untranslated region of human H-ras mRNA and inhibits H-Ras expression, was developed as a potential treatment for cancer, particularly cancers with abnormalities of Ras function [14]. Based on the evidence of the safety, potential effectiveness and feasibility of ISIS-2503 for cancer patients in phase I trials and of the rational approach of combining ISIS-2503 with gemcitabine, a conventional chemotherapy, to obtain a more effective regimen for pancreatic cancer $[15,16]$, a multicenter phase II trial of the combination of ISIS-2503 and gemcitabine in patients with metastatic or locally advanced pancreatic cancer was performed through the North Central Cancer Treatment Group [17]. The clinical study enrolled 48 pancreatic cancer patients, who showed a 6-month survival percentage of $57.5 \%$. The median survival was 6.6 months, and the response rate was $10.4 \%$. The combined regimen was tolerable but insufficient.

The transforming growth factor-beta (TGF- $\beta$ ) signaling pathway is a key player in tumor progression [18], and it was proven that TGF- $\beta 2$ plays a pivotal role in the malignancy and progression of pancreatic cancer [19, 20]. OT-101, an 18-mer phosphorothioate ASO, was designed for the targeted inhibition of human TGF- $\beta 2$ mRNA, and OT-101 suppressed TGF- $\beta 2$ secretion in human pancreatic cancer cell lines [21-23]. Then, a phase I/II clinical trial of OT-101 in 37 patients with advanced pancreatic cancer that showed a good outcome in terms of improved overall survival (OS) was presented at the Gastrointestinal Cancers Symposium in 2017. Moreover, the same group subsequently reported that the levels of IL-8 and IL-15 were positively associated with the OS of pancreatic cancer patients and served as potential predictive biomarkers for the therapeutic effect of combined OT-101 and chemotherapy administration for pancreatic cancer [24].

Signal transducer and activator of transcription (STAT) proteins are a family of cytoplasmic transcription factors that are thought to be candidates for anti-cancer therapeutic options because of the higher dependency of cancer cells than of normal cells on STAT activity [25]. Hong's group showed that AZD9150, a STAT3-inhibiting ASO, had single-agent pharmacologic tumor-suppressive activity in models of human lymphoma and lung cancer [26]. They additionally reported the evaluation of AZD9150 in a non-Hodgkin's lymphoma population [27]. A different group revealed the value of AZD9150 in combination with conventional chemotherapy for patients with neuroblastoma [28]. Programmed cell-death 1 ligand 1 (PD-L1) is a protein of the B7/CD28 family that controls T-cell activation, and MEDI4736, an antagonistic anti-PD- 
L1 monoclonal antibody, may be a promising therapeutic approach for the treatment of cancer [29]. Based on these studies, Hong's group is now recruiting study patients to investigate whether AZD9150 given in combination with MEDI4736 can control advanced pancreatic, lung, or colorectal cancer in a phase II clinical trial (NCT02983578). $\mathrm{X}$-linked inhibitor of apoptosis (XIAP), an endogenous caspase inhibitor, blocks the execution of apoptosis and represents an important survival factor in cancer cells [30]. AEG-35156, a 19-mer phosphorothioate ASO targeting XIAP, was developed for the potential treatment of cancer by lowering the apoptotic threshold and inducing cell death and/or enhancing the cytotoxic action of chemotherapeutic agents [31]. A preclinical study indicated that AEG35156 enhanced the sensitization of tumor necrosis factor-related apoptosis-inducing ligand-mediated apoptosis in pancreatic carcinoma cells, Panc1 [32]. Then, a phase I trial of AEG35156 plus standard-dose gemcitabine in 14 patients with advanced pancreatic cancer was conducted and showed good tolerability but no better results than with gemcitabine alone [33]. Heat shock protein 27 (Hsp27) is a chaperone implicated in several pathological processes, and Hsp27 expression becomes highly upregulated and is also related to higher resistibility to gemcitabine in patients with pancreatic cancer [34, 35]. A modified ASO that is complementary to Hsp27 (OGX-427) has been developed, showing inhibition of tumor progression and enhancement of gemcitabine's efficacy in pancreatic cancer [36]. Thereafter, a phase II trial of OGX-427 plus gemcitabine and nab-paclitaxel in patients with metastatic pancreatic cancer was carried out and revealed that the addition of OGX-427 to chemotherapy had no improved effect in the first-line setting [37]. Thus, clinical trials of the ASO strategy, as a new therapy combined with conventional drugs for advanced states of pancreatic cancer, have not shown significant additional value for these patients, going against our expectations.

\section{RNA interference}

RNAi relies on sequence complementarity between RNAs and the target mRNAs to inhibit their activity. In particular, chemically synthesized siRNAs are mainly used as a new class of therapeutic agents. The siRNA-based therapeutic strategy enables the selective silencing of targeted gene expression. Compared to conventional anti-cancer drugs, siRNA has marked advantages, such as higher safety, higher potency, higher specificity and unrestricted choice of drug targets [38]. As a potent modality of silencing specific the expression of genes that contribute to tumorigenesis and poor prognosis in cancer [39], RNAi-mediated therapeutic intervention can be directed against pancreatic cancer through various pathways [40]. 
It was previously shown that protein kinase N3 (PKN3), a molecule distantly related to protein kinase $\mathrm{C}$, was regulated by the phosphoinositide-3-kinase (PI3K) signal transduction pathway [41]. A preclinical study on Atu027, a liposomal siRNA molecule specifically impeding PKN3 expression in an orthotopic mouse model, underlies the further development of Atu027 as a novel siRNA formulation to block the progression of multiple solid cancers, including pancreatic cancer [42]. A first-in-human phase I trial of Atu027 confirmed its safety in 4 patients with advanced pancreatic cancer [43]. Subsequently, a clinical trial of Atu027 plus gemcitabine in unresectable pancreatic cancer (NCT01808638) was planned in the NIH records, but there has been no report regarding the study yet.

Oncogenic KRAS mutation, an early event in the development of pancreatic cancer, is involved in over $90 \%$ of pancreatic cancer cases [44, 45]. In addition, pancreatic cancer cell growth is dependent on mutated KRAS activity [46]. Accordingly, silencing KRAS is indicative of the inhibition of pancreatic cancer proliferation [47]. On the basis of the above results, Zorde Khvalevsky et al. engineered the anti-KRAS ${ }^{\mathrm{G} 12 \mathrm{D}}$ siRNA (siG12D LODER $^{\mathrm{TM}}$ ) and demonstrated that LODER-derived siG12D significantly suppressed pancreatic cancer growth both in vitro and in vivo and impeded orthotopic pancreatic tumor growth [48], although all of the past studies with direct KRAS modifications for pancreatic cancer failed [49]. As siG12D LODER ${ }^{\mathrm{TM}}$ was designed to be directly implanted into pancreatic cancer under endoscopic ultrasonography (EUS) guidance, this strategy could resolve both the delivery problems associated with RNAi and the impermeable nature of the tumor microenvironment $[50,51]$. In addition, Golan and colleagues performed an open-label phase I/IIa clinical trial and revealed that the combination of siG12D LODER ${ }^{\mathrm{TM}}$ and gemcitabine was well tolerated and safe and had potential value in 15 patients with locally advanced pancreatic cancer, as the median OS was 15.1 months and the 18-month survival was 38.5\% [52]. Subsequently, the biocompatibility regarding the safety and toxicity of siG12D LODER as a local and prolonged delivery system for pancreatic cancer therapy was assessed comprehensively in rats after subcutaneous administration [53]. Further, a phase II clinical trial of siG12D $\mathrm{LODER}^{\mathrm{TM}}$ in combination with gemcitabine plus nab-paclitaxel in patients with locally advanced pancreatic cancer has been ongoing (NCT01676259).

Exosomes, extracellular vesicles generated by all cells, are naturally present in circulating blood. Kamerkar et al. produced exosomes derived from normal fibroblast-like mesenchymal cells for specific delivery of siRNA or shRNA to KRAS ${ }^{\mathrm{G} 12 \mathrm{D}}$ (iExosomes), a common mutation in pancreatic cancer, and showed that iExosomes repressed tumor progression by augmenting the efficacy of exosomes in delivering the therapeutic payload 
in multiple mouse models of pancreatic cancer and significantly improved their OS dependent on CD47-regulated exosome protection from phagocytosis by monocytes and macrophages [54]. In addition, the same group reported on the bioreactor-based largescale production of clinical-grade exosomes derived from bone marrow mesenchymal stem/stromal cells with good manufacturing practice (GMP) grade for promoting the clinical application of iExosome-based therapy [55]. Currently, a phase I study of mesenchymal stromal cell-derived iExosomes for metastatic pancreatic cancer patients harboring the KrasG12D mutation has been planned (NCT03608631).

Recently, complex cancer cell-tumor stroma interactions have been focusing on their crucial roles in tumor initiation, progression, and metastasis [56]. Indeed, medicines targeting the tumor microenvironment, which mediates the therapeutic resistance of pancreatic cancer, have been progressing [57]. It was reported that glycosaminoglycan, a cancer stromal molecule, is involved in various cancer developmental phases, such as proliferation, invasion, metastasis and angiogenesis [4, 58-60]. Specifically, it was found that chondroitin sulfate-E (CS-E), a matrix glycosaminoglycan, was expressed in both the tumor cells and stromal cells surrounding the tumor in pancreatic cancer patient tissues $[59,61]$.

In accord with the above evidence, we previously demonstrated that high CHST15 expression, which is responsible for the biosynthesis of sulfated CS-E, may represent a potential predictive marker of OS in patients with pancreatic cancer following surgical resection [62] and that STNM01, a CHST15 siRNA, successfully inhibited pancreatic tumor growth in xenograft experiments by using the RNAi strategy, as shown in Figure 2 [4]. In addition, in an open-label trial, the safety and feasibility of STNM01 treatment under EUS guided fine-needle injection (EUS-FNI) were certified in six patients with unresectable pancreatic cancer [63]. Based on this accumulating evidence, a clinical phase I/IIa study of STNM01 by using EUS-FNI for patients with unresectable pancreatic cancer obtained approval from the Japan Agency for Medical Research and Development in 2018 .

In short, an RNAi-based strategy for patients with pancreatic cancer is theoretically a prospective therapeutic option; therefore, further studies are anticipated to be translated to the clinic.

\section{MicroRNA}

The recent wide-ranging progression of miRNA-based diagnostic and therapeutic approaches for cancer patients is encouraging and has been widely reported, including in pancreatic cancer [64-84]. Intriguingly, the clinical applications have advanced mainly in 
diagnostic fields instead of treatment options. Indeed, Schultz and colleagues demonstrated the value of serum miRNAs as a diagnostic marker in patients with pancreatic cancer in a case-control study [85], and $\mathrm{Su}$ et al. subsequently identified the serum levels of coexpressed hub miRNAs as potential diagnostic and prognostic biomarkers for pancreatic cancer [86]. In particular, it was reported that circulating microRNA-25 (miR-25) has a high specificity for pancreatic cancer and can be used as a potential biomarker for the early detection of pancreatic cancer based on the comparative data of miR-25 with CEA and CA19-9, which are widely accepted, conventional tumor markers of pancreatic cancer [87, 88]. Based on previous studies, a clinical trial of miR25 for pancreatic cancer by using a detection kit has been planned in China. If the efficacy of miR-25 as a diagnostic biomarker of pancreatic cancer will be certainly proven in the trial, the clinical meaning for early detection would be extremely large. The biological properties of miRNAs that enable them to target multiple genes and act as fine tuners of their expression in vivo account for the simultaneous regulation of the related diseasespecific genes in their network. Accordingly, clinical trials of miRNA for pancreatic cancer will probably continue to grow in number, especially in the field of diagnosis.

\section{Aptamers}

Aptamers are single-stranded oligonucleotide ligands that demonstrate high affinity toward target proteins and inhibit their physiological effect by forming complexes with proteins based on their unique three-dimensional folding in the later phase of disease progression [89, 90]. Systematic Evolution of Ligands by Exponential Enrichment (SELEX) is an experimental procedure that enables researchers to choose the optimum aptamer from a vast RNA pool for binding with target proteins [91]. Indeed, it has been reported that aptamers can act as players of various roles, such as oncosuppressors, biomarkers, cargo and detectors, for pancreatic cancer, as shown in Table 2.

Kim and colleagues generated a 2'-fluoropyrimidine-modified RNA aptamer (P12FR2) that binds specifically to human pancreatic adenocarcinoma upregulated factor (PAUF) and showed the oncosuppressive role of P12FR2 both in vitro and in vivo, suggesting that P12FR2 is a potential therapeutic target of human pancreatic cancer [92]. Based on their previous work, PAUF is a novel secretory protein involved in pancreatic cancer progression [93].

Ray et al. devised an aptamer strategy to identify RNA ligands that could detect structural differences between the secretomes of pancreatic cancer cells and noncancerous cells [94]. Within their identification strategy, an aptamer (M9-5) that preferentially bound the pancreatic cancer secretome over the noncancerous secretome 
and targeted cyclophilin B (СypB) was confirmed. The M9-5 binding analysis indicated excellent potential as a serum biomarker to distinguish between pancreatic cancer patients and healthy volunteers by using the M9-5 aptamer [94]. In addition, the authors characterized the aptamer and the target protein, CypB. Their posttranslational modifications on $\mathrm{CypB}$ could discriminate $\mathrm{CypB}$ expressed in human cells versus bacteria, and their findings accelerate the use of aptamers as important biomarkers [95]. Further, the same group reported a different targeted aptamer. Based on the evidence that most pancreatic cancers overexpress epidermal growth factor receptor (EGFR), a transmembrane receptor tyrosine kinase [96], they generated a nuclease-resistant RNA aptamer and found that the aptamer was internalized by EGFR on human pancreatic cancer cells by efficiently delivering gemcitabine-containing polymers into EGFRexpressing cells, resulting in the inhibition of the proliferation of pancreatic cancer cells in vitro [97]. Their work supported the value of the aptamer strategy in that the targeted delivery of active metabolites into cancer cells could effectively overcome chemoresistance and minimize toxic effects by decreasing uptake into normal cells. Several studies have used SELEX aptamers for pancreatic cancer. Zhang et al. identified the usability of a SELEX aptamer (BC-15) as a detector of circulating tumor cells in the peripheral blood of patients with pancreatic cancer [98]. A different group focused on DNA aptamers and showed potential efficacy for pancreatic cancer. They successfully generated eight aptamers with high affinity for pancreatic ductal adenocarcinoma by using cell-SELEX targeting a human pancreatic ductal adenocarcinoma cell line, PL45 [99]. They further developed a truncated DNA aptamer, termed XQ-2d, and proved its high affinity and specificity against the PL45 pancreatic cancer cell line, with a detection ratio of $82.5 \%$ [100]. Their work might be a meaningful step in the development of probes as molecular imaging tools for the early detection of pancreatic cancer.

Targeted delivery by means of aptamers of effective treatment molecules directly to pancreatic cancer cells might maximize the therapeutic value and minimize the possible adverse events by decreasing uptake into normal biological cells. A study showed that a pancreatic cancer-specific RNA aptamer can be used for the targeted delivery of the nucleoside drug 5-fluoro-2'-deoxyuridine to pancreatic cancer cells expressing alkaline phosphatase placental-like 2, a putative biomarker, leading to the inhibition of tumor growth [101].

Yoon and colleagues successfully developed a novel aptamer-based method of delivering targeted molecular therapy in pancreatic cancer by upregulating the transcription factor CCAAT/enhancer-binding protein- $\alpha(\mathrm{C} / \mathrm{EBP} \alpha)$, owing to its binding 
with pancreatic cancer-specific 2'-fluropyrimidine RNA aptamers P19 and P1, which were selected by SELEX, resulting in tumor-suppressive effects [102]. Subsequently, their group made further improvements in chemotherapeutic efficacy for pancreatic cancer by forming hybridized aptamer-drug conjugates that involved pancreatic cancerspecific RNA aptamer P19, conducive to higher selectivity of drug delivery with a less toxic effect [103]. Impressively, they proposed a different therapeutic conjugation of pancreatic cancer-specific 2'-fluoropyrimidine RNA-aptamers and C/EBP $\alpha$-small activating RNA, which accounted for the inhibition of pancreatic cancer growth [104]. Their works suggest that diverse aptamer strategies can be applied for the treatment of pancreatic cancer by focusing on the outstanding targeted delivery potency. In contrast, Kratschmer et al. provided a direct conjugation strategy by generating small-molecule aptamer-toxin conjugates of auristatin, monomethyl auristatin E (MMAE) and monomethyl auristatin F (MMAF), and confirmed the improved cytotoxicity of MMAE and MMAF conjugated to an anti-transferrin receptor aptamer and an anti-EGFR aptamer in three different human pancreatic cancer cell lines [105].

Based on the evidence that the $G$ protein-coupled receptor cholecystokinin B receptor (CCKBR) is constitutively overexpressed on the surface of human pancreatic cancer cells and plays a role in tumor cell proliferation [106], Clawson et al. identified highaffinity DNA aptamers to the CCKBR and revealed their characterization and targeted efficacy of delivering diagnostic agents or therapeutic cargos to pancreatic cancer cells with minimal side effects by using dual SELEX selection from a pool of multiple DNA aptamers [107]. A Korean group also assessed the potential of pancreatic cancer stem cell-associated aptamers as a diagnostic marker and therapeutic option with a modified SELEX method [108]. The most recent study showed the therapeutic effects on pancreatic cancer cells in vitro and in vivo by constructing an aptamer-based gemcitabine delivery system, APTA-12 [109]. Altogether, the aptamer strategies have shown promising advances as diagnostic or therapeutic options for pancreatic cancer, mainly in the field of targeted drug delivery to cancerous lesions based on the benefits of the SELEX procedure; thus, it seems that a clinical trial of aptamer oligonucleotides for patients with pancreatic cancer should be planned in the near future.

\section{Decoys}

A decoy, which forms a double-stranded DNA structure, inhibits DNA transcription at the initial phase of disease progression. Compared with the research of other oligonucleotides, it seems that the research progress on decoys for pancreatic cancer has 
been delayed. Regarding the utilized methods of decoy for pancreatic cancer, only one therapeutic and two delivery methods have been reported.

Cogoi et al. focused on myc-associated zinc-finger (MAZ), which is a G4-DNA structure that binds to nuclear proteins to promote the activation of Kras transcription. It is well known that the Kras mutation is a primary trigger in $>90 \%$ of cases of pancreatic cancer progression [110-112] and contains a nuclear-hypersensitive element (NHE). Therefore, the authors designed oligonucleotides that mimicked one of the Gquadruplexes formed by NHE (G4-decoys) and found that the decoy strategy inhibits Kras in pancreatic cancer cells and reduces tumor growth in human pancreatic cancer xenograft mice [113]. Moreover, the authors subsequently reported a lipid-modified strategy based on the use of palmitoyl-oleyl-phosphatidylcholine liposomes, which improved the delivery of the G4-decoy by coupling to the liposomes efficiently, resulting in decreased Kras transcription levels and metabolic activity of pancreatic cancer cells [114].

Based on a previous study in which high-mobility group AT-hook 1 (HMGA1) promotes chemoresistance to gemcitabine through an Akt-dependent mechanism in human pancreatic cancer cells [115], Hassan et al. introduced a new strategy of delivering decoy HMGA1-hyperbinding sites into the nucleus of pancreatic cancer cells using an engineered adenovirus, leading to the sequestration of excess amounts of nuclear HMGA1 with the decoy hyperbinding sites and resulting in the reduction of oncogenic pancreatic cells and the HMGA1-associated gemcitabine resistance [116]. Taken together, the decoy strategies are expected to be translated into various clinical applications as a new cancer treatment to specifically target any overexpressed oncogenic transcription factor that binds double-stranded DNA.

Given the small number of preclinical studies with decoy oligonucleotides for pancreatic cancer, there is still ample room for further development of clinical applications.

\section{Conclusions}

Recent advances in oligonucleotide therapeutics with specific inhibition of targeted gene expression have been astonishing, especially in the cancer field. Thus, there is no doubt that this class of therapeutics is close to becoming a breakthrough cancer treatment. To respond to the urgent need for new crucial drugs for advanced pancreatic cancer, further studies on oligonucleotides are still needed to improve their quality and clinical availability, which should lead to a new definitive treatment against refractory disease. 
Author Contributions: Writing-Original Draft Preparation, K.T.; Writing-Review and Editing, A.K., Y.T.; Supervision, S.K., N.Y. and M.S. All authors have read and approved the final manuscript.

Funding: This research received no external funding.

Conflicts of Interest: The authors declare no conflict of interest.

$\begin{array}{ll}\text { Abbreviations } & \\ \text { ASO } & \text { antisense oligonucleotide } \\ \text { CCKBR } & \text { cholecystokinin B receptor } \\ \text { C/EBP } \alpha & \text { CCAAT/enhancer-binding protein- } \alpha \\ \text { CHST15 } & \text { carbohydrate sulfotransferase15 } \\ \text { CS-E } & \text { chondroitin sulfate-E } \\ \text { CypB } & \text { cyclophilin B } \\ \text { DNA } & \text { deoxyribonucleic acid } \\ \text { EGFR } & \text { epidermal growth factor receptor } \\ \text { EUS } & \text { endoscopic ultrasonography } \\ \text { EUS-FNI } & \text { endoscopic ultrasonography guided fine-needle injection } \\ \text { GMP } & \text { good manufacturing practice } \\ \text { HIV-1 } & \text { human immunodeficiency virus-1 } \\ \text { HMGA1 } & \text { high mobility group AT-hook 1 } \\ \text { MAZ } & \text { myc-associated zinc-finger } \\ \text { miRNA } & \text { micro RNA } \\ \text { MMAE } & \text { monomethyl auristatin E } \\ \text { MMAF } & \text { monomethyl auristatin F } \\ \text { NHE } & \text { nuclear-hypersensitive element } \\ \text { NIH } & \text { National Institutes of Health } \\ \text { OS } & \text { overall survival } \\ \text { PAUF } & \text { pancreatic adenocarcinoma upregulated factor } \\ \end{array}$


PD-L1 programmed cell-death 1 ligand 1

P12FR2 -fluoropyrimidine modified RNA aptamer

RNA ribonucleic acid

RNAi RNA interference

RSV respiratory syncytial virus

SELEX Systematic Evolution of Ligands by Exponential Enrichment siRNA small interfering RNA

STAT signal transducer and activator of transcription

TGF- $\beta \quad$ transforming growth factor-beta

XIAP X-linked inhibitor of apoptosis 


\section{References}

1. Scherman, D.; Rousseau, A.; Bigey, P.; Escriou, V., Genetic pharmacology: progresses in siRNA delivery and therapeutic applications. Gene therapy 2017, 24, 151.

2. Aier, I.; Semwal, R.; Sharma, A.; Varadwaj, P. K., A systematic assessment of statistics, risk factors, and underlying features involved in pancreatic cancer. Cancer epidemiology 2019, 58, 104-110.

3. McGuigan, A.; Kelly, P.; Turkington, R. C.; Jones, C.; Coleman, H. G.; McCain, R. S., Pancreatic cancer: A review of clinical diagnosis, epidemiology, treatment and outcomes. World journal of gastroenterology: WJG 2018, 24, (43), 4846-4861.

4. Takakura, K.; Shibazaki, Y.; Yoneyama, H.; Fujii, M.; Hashiguchi, T.; Ito, Z.; Kajihara, M.; Misawa, T.; Homma, S.; Ohkusa, T.; Koido, S., Inhibition of Cell Proliferation and Growth of Pancreatic Cancer by Silencing of Carbohydrate Sulfotransferase 15 In Vitro and in a Xenograft Model. PloS one 2015, 10, (12), e0142981.

5. Belikova, A. M.; Zarytova, V. F.; Grineva, N. I., Synthesis of ribonucleosides and diribonucleoside phosphates containing 2-chloroethylamine and nitrogen mustard residues. Tetrahedron letters 1967, 37, 3557-62.

6. Zamecnik, P. C.; Stephenson, M. L., Inhibition of Rous sarcoma virus replication and cell transformation by a specific oligodeoxynucleotide. Proceedings of the National Academy of Sciences of the United States of America 1978, 75, (1), 280-4.

7. Rapaport, E.; Zamecnik, P. C., Increased incorporation of adenosine into adenine nucleotide pools in serum-deprived mammalian cells. Proceedings of the National Academy of Sciences of the United States of America 1978, 75, (3), 1145-7.

8. Matsukura, M.; Zon, G.; Shinozuka, K.; Robert-Guroff, M.; Shimada, T.; Stein, C. A.; Mitsuya, H.; Wong-Staal, F.; Cohen, J. S.; Broder, S., Regulation of viral expression of human immunodeficiency virus in vitro by an antisense phosphorothioate oligodeoxynucleotide against rev (art/trs) in chronically infected cells. Proceedings of the National Academy of Sciences of the United States of America 1989, 86, (11), 4244-8.

9. Cullen, B. R.; Greene, W. C., Regulatory pathways governing HIV-1 replication. Cell 1989, 58, (3), 423-6.

10. Fire, A.; Xu, S.; Montgomery, M. K.; Kostas, S. A.; Driver, S. E.; Mello, C. C., Potent and specific genetic interference by double-stranded RNA in Caenorhabditis elegans. Nature 1998, 391, (6669), 806-11. 
11. DeVincenzo, J.; Cehelsky, J. E.; Alvarez, R.; Elbashir, S.; Harborth, J.; Toudjarska, I.; Nechev, L.; Murugaiah, V.; Van Vliet, A.; Vaishnaw, A. K.; Meyers, R., Evaluation of the safety, tolerability and pharmacokinetics of ALN-RSV01, a novel RNAi antiviral therapeutic directed against respiratory syncytial virus (RSV). Antiviral research 2008, 77, (3), 225-31.

12. Almeida, M. I.; Reis, R. M.; Calin, G. A., MicroRNA history: discovery, recent applications, and next frontiers. Mutation research 2011, 717, (1-2), 1-8.

13. Kohn, D. B.; Bauer, G.; Rice, C. R.; Rothschild, J. C.; Carbonaro, D. A.; Valdez, P.; Hao, Q.; Zhou, C.; Bahner, I.; Kearns, K.; Brody, K.; Fox, S.; Haden, E.; Wilson, K.; Salata, C.; Dolan, C.; Wetter, C.; Aguilar-Cordova, E.; Church, J., A clinical trial of retroviral-mediated transfer of a rev-responsive element decoy gene into CD34(+) cells from the bone marrow of human immunodeficiency virus-1-infected children. Blood 1999, 94, (1), 368-71.

14. Monia, B. P.; Johnston, J. F.; Ecker, D. J.; Zounes, M. A.; Lima, W. F.; Freier, S. M., Selective inhibition of mutant Ha-ras mRNA expression by antisense oligonucleotides. The Journal of biological chemistry 1992, 267, (28), 19954-62.

15. Cunningham, C. C.; Holmlund, J. T.; Geary, R. S.; Kwoh, T. J.; Dorr, A.; Johnston, J. F.; Monia, B.; Nemunaitis, J., A Phase I trial of H-ras antisense oligonucleotide ISIS 2503 administered as a continuous intravenous infusion in patients with advanced carcinoma. Cancer 2001, 92, (5), 1265-71.

16. Adjei, A. A.; Dy, G. K.; Erlichman, C.; Reid, J. M.; Sloan, J. A.; Pitot, H. C.; Alberts, S. R.; Goldberg, R. M.; Hanson, L. J.; Atherton, P. J.; Watanabe, T.; Geary, R. S.; Holmlund, J.; Dorr, F. A., A phase I trial of ISIS 2503, an antisense inhibitor of Hras, in combination with gemcitabine in patients with advanced cancer. Clin Cancer Res 2003, 9, (1), 115-23.

17. Alberts, S. R.; Schroeder, M.; Erlichman, C.; Steen, P. D.; Foster, N. R.; Moore, D. F., Jr.; Rowland, K. M., Jr.; Nair, S.; Tschetter, L. K.; Fitch, T. R., Gemcitabine and ISIS-2503 for patients with locally advanced or metastatic pancreatic adenocarcinoma: a North Central Cancer Treatment Group phase II trial. J Clin Oncol 2004, 22, (24), 4944-50.

18. Massague, J., TGFbeta in Cancer. Cell 2008, 134, (2), 215-30.

19. Friess, H.; Yamanaka, Y.; Buchler, M.; Ebert, M.; Beger, H. G.; Gold, L. I.; Korc, M., Enhanced expression of transforming growth factor beta isoforms in pancreatic cancer correlates with decreased survival. Gastroenterology 1993, 105, (6), 1846-56.

20. von Bernstorff, W.; Voss, M.; Freichel, S.; Schmid, A.; Vogel, I.; Johnk, C.; HenneBruns, D.; Kremer, B.; Kalthoff, H., Systemic and local immunosuppression in 
pancreatic cancer patients. Clin Cancer Res 2001, 7, (3 Suppl), 925s-932s.

21. Schlingensiepen, K. H.; Schlingensiepen, R.; Steinbrecher, A.; Hau, P.; Bogdahn, U.; Fischer-Blass, B.; Jachimczak, P., Targeted tumor therapy with the TGF-beta 2 antisense compound AP 12009. Cytokine \& growth factor reviews 2006, 17, (1-2), 129-39.

22. Jaschinski, F.; Rothhammer, T.; Jachimczak, P.; Seitz, C.; Schneider, A.; Schlingensiepen, K. H., The antisense oligonucleotide trabedersen (AP 12009) for the targeted inhibition of TGF-beta2. Current pharmaceutical biotechnology 2011, $12,(12), 2203-13$.

23. Schlingensiepen, K. H.; Jaschinski, F.; Lang, S. A.; Moser, C.; Geissler, E. K.; Schlitt, H. J.; Kielmanowicz, M.; Schneider, A., Transforming growth factor-beta 2 gene silencing with trabedersen (AP 12009) in pancreatic cancer. Cancer science 2011, 102, (6), 1193-200.

24. D'Cruz, O. J.; Qazi, S.; Hwang, L.; Ng, K.; Trieu, V., Impact of targeting transforming growth factor beta-2 with antisense OT-101 on the cytokine and chemokine profile in patients with advanced pancreatic cancer. OncoTargets and therapy 2018, 11, 2779-2796.

25. Furqan, M.; Akinleye, A.; Mukhi, N.; Mittal, V.; Chen, Y.; Liu, D., STAT inhibitors for cancer therapy. Journal of hematology \& oncology 2013, 6, 90.

26. Hong, D.; Kurzrock, R.; Kim, Y.; Woessner, R.; Younes, A.; Nemunaitis, J.; Fowler, N.; Zhou, T.; Schmidt, J.; Jo, M.; Lee, S. J.; Yamashita, M.; Hughes, S. G.; Fayad, L.; Piha-Paul, S.; Nadella, M. V.; Mohseni, M.; Lawson, D.; Reimer, C.; Blakey, D. C.; Xiao, X.; Hsu, J.; Revenko, A.; Monia, B. P.; MacLeod, A. R., AZD9150, a nextgeneration antisense oligonucleotide inhibitor of STAT3 with early evidence of clinical activity in lymphoma and lung cancer. Science translational medicine 2015, 7, (314), 314ra185.

27. Reilley, M. J.; McCoon, P.; Cook, C.; Lyne, P.; Kurzrock, R.; Kim, Y.; Woessner, R.; Younes, A.; Nemunaitis, J.; Fowler, N.; Curran, M.; Liu, Q.; Zhou, T.; Schmidt, J.; Jo, M.; Lee, S. J.; Yamashita, M.; Hughes, S. G.; Fayad, L.; Piha-Paul, S.; Nadella, M. V. P.; Xiao, X.; Hsu, J.; Revenko, A.; Monia, B. P.; MacLeod, A. R.; Hong, D. S., STAT3 antisense oligonucleotide AZD9150 in a subset of patients with heavily pretreated lymphoma: results of a phase $1 \mathrm{~b}$ trial. Journal for immunotherapy of cancer 2018, 6, (1), 119.

28. Odate, S.; Veschi, V.; Yan, S.; Lam, N.; Woessner, R.; Thiele, C. J., Inhibition of STAT3 with the Generation 2.5 Antisense Oligonucleotide, AZD9150, Decreases Neuroblastoma Tumorigenicity and Increases Chemosensitivity. Clin Cancer Res 
2017, 23, (7), 1771-1784.

29. Stewart, R.; Morrow, M.; Hammond, S. A.; Mulgrew, K.; Marcus, D.; Poon, E.; Watkins, A.; Mullins, S.; Chodorge, M.; Andrews, J.; Bannister, D.; Dick, E.; Crawford, N.; Parmentier, J.; Alimzhanov, M.; Babcook, J. S.; Foltz, I. N.; Buchanan, A.; Bedian, V.; Wilkinson, R. W.; McCourt, M., Identification and Characterization of MEDI4736, an Antagonistic Anti-PD-L1 Monoclonal Antibody. Cancer immunology research 2015, 3, (9), 1052-62.

30. Cheung, H. H.; LaCasse, E. C.; Korneluk, R. G., X-linked inhibitor of apoptosis antagonism: strategies in cancer treatment. Clin Cancer Res 2006, 12, (11 Pt 1), 3238-42.

31. Lacasse, E. C.; Kandimalla, E. R.; Winocour, P.; Sullivan, T.; Agrawal, S.; Gillard, J. W.; Durkin, J., Application of XIAP antisense to cancer and other proliferative disorders: development of AEG35156/ GEM640. Annals of the New York Academy of Sciences 2005, 1058, 215-34.

32. LaCasse, E. C.; Cherton-Horvat, G. G.; Hewitt, K. E.; Jerome, L. J.; Morris, S. J.; Kandimalla, E. R.; Yu, D.; Wang, H.; Wang, W.; Zhang, R.; Agrawal, S.; Gillard, J. W.; Durkin, J. P., Preclinical characterization of AEG35156/GEM 640, a secondgeneration antisense oligonucleotide targeting X-linked inhibitor of apoptosis. Clin Cancer Res 2006, 12, (17), 5231-41.

33. Mahadevan, D.; Chalasani, P.; Rensvold, D.; Kurtin, S.; Pretzinger, C.; Jolivet, J.; Ramanathan, R. K.; Von Hoff, D. D.; Weiss, G. J., Phase I trial of AEG35156 an antisense oligonucleotide to XIAP plus gemcitabine in patients with metastatic pancreatic ductal adenocarcinoma. American journal of clinical oncology 2013, 36, (3), 239-43.

34. Melle, C.; Ernst, G.; Escher, N.; Hartmann, D.; Schimmel, B.; Bleul, A.; Thieme, H.; Kaufmann, R.; Felix, K.; Friess, H. M.; Settmacher, U.; Hommann, M.; Richter, K. K.; Daffner, W.; Taubig, H.; Manger, T.; Claussen, U.; von Eggeling, F., Protein profiling of microdissected pancreas carcinoma and identification of HSP27 as a potential serum marker. Clinical chemistry 2007, 53, (4), 629-35.

35. Mori-Iwamoto, S.; Kuramitsu, Y.; Ryozawa, S.; Mikuria, K.; Fujimoto, M.; Maehara, S.; Maehara, Y.; Okita, K.; Nakamura, K.; Sakaida, I., Proteomics finding heat shock protein 27 as a biomarker for resistance of pancreatic cancer cells to gemcitabine. International journal of oncology 2007, 31, (6), 1345-50.

36. Baylot, V.; Andrieu, C.; Katsogiannou, M.; Taieb, D.; Garcia, S.; Giusiano, S.; Acunzo, J.; Iovanna, J.; Gleave, M.; Garrido, C.; Rocchi, P., OGX-427 inhibits tumor progression and enhances gemcitabine chemotherapy in pancreatic cancer. Cell 
death \& disease 2011,2 , e221.

37. Ko, A. H.; Murphy, P. B.; Peyton, J. D.; Shipley, D. L.; Al-Hazzouri, A.; Rodriguez, F. A.; Womack, M. S. t.; Xiong, H. Q.; Waterhouse, D. M.; Tempero, M. A.; Guo, S.; Lane, C. M.; Earwood, C.; DeBusk, L. M.; Bendell, J. C., A Randomized, DoubleBlinded, Phase II Trial of Gemcitabine and Nab-Paclitaxel Plus Apatorsen or Placebo in Patients with Metastatic Pancreatic Cancer: The RAINIER Trial. Oncologist 2017, 22, (12), 1427-e129.

38. Zuckerman, J. E.; Davis, M. E., Clinical experiences with systemically administered siRNA-based therapeutics in cancer. Nature reviews. Drug discovery 2015, 14, (12), 843-56.

39. Kim, D. H.; Rossi, J. J., Strategies for silencing human disease using RNA interference. Nature reviews. Genetics 2007, 8, (3), 173-84.

40. Chang, H., RNAi-mediated knockdown of target genes: a promising strategy for pancreatic cancer research. Cancer gene therapy 2007, 14, (8), 677-85.

41. Leenders, F.; Mopert, K.; Schmiedeknecht, A.; Santel, A.; Czauderna, F.; Aleku, M.; Penschuck, S.; Dames, S.; Sternberger, M.; Rohl, T.; Wellmann, A.; Arnold, W.; Giese, K.; Kaufmann, J.; Klippel, A., PKN3 is required for malignant prostate cell growth downstream of activated PI 3-kinase. The EMBO journal 2004, 23, (16), 3303-13.

42. Aleku, M.; Schulz, P.; Keil, O.; Santel, A.; Schaeper, U.; Dieckhoff, B.; Janke, O.; Endruschat, J.; Durieux, B.; Roder, N.; Loffler, K.; Lange, C.; Fechtner, M.; Mopert, K.; Fisch, G.; Dames, S.; Arnold, W.; Jochims, K.; Giese, K.; Wiedenmann, B.; Scholz, A.; Kaufmann, J., Atu027, a liposomal small interfering RNA formulation targeting protein kinase N3, inhibits cancer progression. Cancer Res 2008, 68, (23), 9788-98.

43. Schultheis, B.; Strumberg, D.; Santel, A.; Vank, C.; Gebhardt, F.; Keil, O.; Lange, C.; Giese, K.; Kaufmann, J.; Khan, M.; Drevs, J., First-in-human phase I study of the liposomal RNA interference therapeutic Atu027 in patients with advanced solid tumors. J Clin Oncol 2014, 32, (36), 4141-8.

44. Belda-Iniesta, C.; Ibanez de Caceres, I.; Barriuso, J.; de Castro Carpeno, J.; Gonzalez Baron, M.; Feliu, J., Molecular biology of pancreatic cancer. Clinical \& translational oncology : official publication of the Federation of Spanish Oncology Societies and of the National Cancer Institute of Mexico 2008, 10, (9), 530-7.

45. Feldmann, G.; Beaty, R.; Hruban, R. H.; Maitra, A., Molecular genetics of pancreatic intraepithelial neoplasia. Journal of hepato-biliary-pancreatic surgery 2007, 14, (3), 224-32. 
46. Singh, A.; Greninger, P.; Rhodes, D.; Koopman, L.; Violette, S.; Bardeesy, N.; Settleman, J., A gene expression signature associated with "K-Ras addiction" reveals regulators of EMT and tumor cell survival. Cancer Cell 2009, 15, (6), 489500 .

47. Rejiba, S.; Wack, S.; Aprahamian, M.; Hajri, A., K-ras oncogene silencing strategy reduces tumor growth and enhances gemcitabine chemotherapy efficacy for pancreatic cancer treatment. Cancer science 2007, 98, (7), 1128-36.

48. Zorde Khvalevsky, E.; Gabai, R.; Rachmut, I. H.; Horwitz, E.; Brunschwig, Z.; Orbach, A.; Shemi, A.; Golan, T.; Domb, A. J.; Yavin, E.; Giladi, H.; Rivkin, L.; Simerzin, A.; Eliakim, R.; Khalaileh, A.; Hubert, A.; Lahav, M.; Kopelman, Y.; Goldin, E.; Dancour, A.; Hants, Y.; Arbel-Alon, S.; Abramovitch, R.; Shemi, A.; Galun, E., Mutant KRAS is a druggable target for pancreatic cancer. Proceedings of the National Academy of Sciences of the United States of America 2013, 110, (51), 20723-8.

49. Eser, S.; Schnieke, A.; Schneider, G.; Saur, D., Oncogenic KRAS signalling in pancreatic cancer. British journal of cancer 2014, 111, (5), 817-22.

50. Xu, Z.; Wang, Y.; Zhang, L.; Huang, L., Nanoparticle-delivered transforming growth factor-beta siRNA enhances vaccination against advanced melanoma by modifying tumor microenvironment. ACS nano 2014, 8, (4), 3636-45.

51. Jain, R. K., Transport of molecules, particles, and cells in solid tumors. Annual review of biomedical engineering 1999, 1, 241-63.

52. Golan, T.; Khvalevsky, E. Z.; Hubert, A.; Gabai, R. M.; Hen, N.; Segal, A.; Domb, A.; Harari, G.; David, E. B.; Raskin, S.; Goldes, Y.; Goldin, E.; Eliakim, R.; Lahav, M.; Kopleman, Y.; Dancour, A.; Shemi, A.; Galun, E., RNAi therapy targeting KRAS in combination with chemotherapy for locally advanced pancreatic cancer patients. Oncotarget 2015, 6, (27), 24560-70.

53. Ramot, Y.; Rotkopf, S.; Gabai, R. M.; Zorde Khvalevsky, E.; Muravnik, S.; Marzoli, G. A.; Domb, A. J.; Shemi, A.; Nyska, A., Preclinical Safety Evaluation in Rats of a Polymeric Matrix Containing an siRNA Drug Used as a Local and Prolonged Delivery System for Pancreatic Cancer Therapy. Toxicologic pathology 2016, 44, (6), 856-65.

54. Kamerkar, S.; LeBleu, V. S.; Sugimoto, H.; Yang, S.; Ruivo, C. F.; Melo, S. A.; Lee, J. J.; Kalluri, R., Exosomes facilitate therapeutic targeting of oncogenic KRAS in pancreatic cancer. Nature 2017, 546, (7659), 498-503.

55. Mendt, M.; Kamerkar, S.; Sugimoto, H.; McAndrews, K. M.; Wu, C. C.; Gagea, M.; Yang, S.; Blanko, E. V. R.; Peng, Q.; Ma, X.; Marszalek, J. R.; Maitra, A.; Yee, C.; 
Rezvani, K.; Shpall, E.; LeBleu, V. S.; Kalluri, R., Generation and testing of clinicalgrade exosomes for pancreatic cancer. JCI insight 2018, 3, (8).

56. Valkenburg, K. C.; de Groot, A. E.; Pienta, K. J., Targeting the tumour stroma to improve cancer therapy. Nature reviews. Clinical oncology 2018, 15, (6), 366-381.

57. Neesse, A.; Krug, S.; Gress, T. M.; Tuveson, D. A.; Michl, P., Emerging concepts in pancreatic cancer medicine: targeting the tumor stroma. OncoTargets and therapy 2013, 7, 33-43.

58. Fuster, M. M.; Esko, J. D., The sweet and sour of cancer: glycans as novel therapeutic targets. Nat Rev Cancer 2005, 5, (7), 526-42.

59. Sugahara, K. N.; Hirata, T.; Tanaka, T.; Ogino, S.; Takeda, M.; Terasawa, H.; Shimada, I.; Tamura, J.; ten Dam, G. B.; van Kuppevelt, T. H.; Miyasaka, M., Chondroitin sulfate E fragments enhance CD44 cleavage and CD44-dependent motility in tumor cells. Cancer Res 2008, 68, (17), 7191-9.

60. Mizumoto, S.; Sugahara, K., Glycosaminoglycans are functional ligands for receptor for advanced glycation end-products in tumors. The FEBS journal 2013, 280, (10), 2462-70.

61. Sugahara, K.; Mikami, T.; Uyama, T.; Mizuguchi, S.; Nomura, K.; Kitagawa, H., Recent advances in the structural biology of chondroitin sulfate and dermatan sulfate. Current opinion in structural biology 2003, 13, (5), 612-20.

62. Ito, Z.; Takakura, K.; Suka, M.; Kanai, T.; Saito, R.; Fujioka, S.; Kajihara, M.; Yanagisawa, H.; Misawa, T.; Akiba, T.; Koido, S.; Ohkusa, T., Prognostic impact of carbohydrate sulfotransferase 15 in patients with pancreatic ductal adenocarcinoma. Oncology letters 2017, 13, (6), 4799-4805.

63. Nishimura, M.; Matsukawa, M.; Fujii, Y.; Matsuda, Y.; Arai, T.; Ochiai, Y.; Itoi, T.; Yahagi, N., Effects of EUS-guided intratumoral injection of oligonucleotide STNM01 on tumor growth, histology, and overall survival in patients with unresectable pancreatic cancer. Gastrointestinal endoscopy 2018, 87, (4), 1126-1131.

64. Takahashi, R. U.; Prieto-Vila, M.; Kohama, I.; Ochiya, T., Development of miRNAbased therapeutic approaches for cancer patients. Cancer science 2019.

65. Gurbuz, N.; Ozpolat, B., MicroRNA-based Targeted Therapeutics in Pancreatic Cancer. Anticancer research 2019, 39, (2), 529-532.

66. Mollaei, H.; Safaralizadeh, R.; Rostami, Z., MicroRNA replacement therapy in cancer. Journal of cellular physiology 2019.

67. Ors-Kumoglu, G.; Gulce-Iz, S.; Biray-Avci, C., Therapeutic microRNAs in human cancer. Cytotechnology 2019, 71, (1), 411-425.

68. Slotwinski, R.; Lech, G.; Slotwinska, S. M., MicroRNAs in pancreatic cancer 
diagnosis and therapy. Central-European journal of immunology 2018, 43, (3), 314324 .

69. Tesfaye, A. A.; Azmi, A. S.; Philip, P. A., miRNA and Gene Expression in Pancreatic Ductal Adenocarcinoma. The American journal of pathology 2019, 189, (1), 58-70.

70. Baradaran, B.; Shahbazi, R.; Khordadmehr, M., Dysregulation of key microRNAs in pancreatic cancer development. Biomedicine \& pharmacotherapy $=$ Biomedecine $\&$ pharmacotherapie 2019, 109, 1008-1015.

71. Biswas, S., MicroRNAs as Therapeutic Agents: The Future of the Battle Against Cancer. Current topics in medicinal chemistry 2018, 18, (30), 2544-2554.

72. Kwok, G. T.; Zhao, J. T.; Weiss, J.; Mugridge, N.; Brahmbhatt, H.; MacDiarmid, J. A.; Robinson, B. G.; Sidhu, S. B., Translational applications of microRNAs in cancer, and therapeutic implications. Non-coding $R N A$ research 2017,2 , (3-4), 143-150.

73. Ghasabi, M.; Mansoori, B.; Mohammadi, A.; Duijf, P. H.; Shomali, N.; Shirafkan, N.; Mokhtarzadeh, A.; Baradaran, B., MicroRNAs in cancer drug resistance: Basic evidence and clinical applications. Journal of cellular physiology 2019, 234, (3), 2152-2168.

74. Dragomir, M.; Mafra, A. C. P.; Dias, S. M. G.; Vasilescu, C.; Calin, G. A., Using microRNA Networks to Understand Cancer. International journal of molecular sciences 2018, 19, (7).

75. Guo, S.; Fesler, A.; Wang, H.; Ju, J., microRNA based prognostic biomarkers in pancreatic Cancer. Biomarker research 2018, 6, 18.

76. Sun, X.; Zhou, X.; Zhang, Y.; Zhu, X.; Liu, H., Systematic Review and Meta-Analysis of Diagnostic Accuracy of miRNAs in Patients with Pancreatic Cancer. Disease markers 2018, 2018, 6292396.

77. Kian, R.; Moradi, S.; Ghorbian, S., Role of components of microRNA machinery in carcinogenesis. Experimental oncology 2018, 40, (1), 2-9.

78. Hosseinahli, N.; Aghapour, M.; Duijf, P. H. G.; Baradaran, B., Treating cancer with microRNA replacement therapy: A literature review. Journal of cellular physiology 2018, 233, (8), 5574-5588.

79. Vannini, I.; Fanini, F.; Fabbri, M., Emerging roles of microRNAs in cancer. Curr Opin Genet Dev 2018, 48, 128-133.

80. Petrovic, N.; Ergun, S., miRNAs as Potential Treatment Targets and Treatment Options in Cancer. Molecular diagnosis \& therapy 2018, 22, (2), 157-168.

81. Nguyen, D. D.; Chang, S., Development of Novel Therapeutic Agents by Inhibition of Oncogenic MicroRNAs. International journal of molecular sciences 2017, 19, (1).

82. Qadir, M. I.; Faheem, A., miRNA: A Diagnostic and Therapeutic Tool for Pancreatic 
Cancer. Critical reviews in eukaryotic gene expression 2017, 27, (3), 197-204.

83. Mansoori, B.; Mohammadi, A.; Shirjang, S.; Baradaran, B., MicroRNAs in the Diagnosis and Treatment of Cancer. Immunological investigations 2017, 46, (8), 880-897.

84. Drusco, A.; Croce, C. M., MicroRNAs and Cancer: A Long Story for Short RNAs. Advances in cancer research 2017, 135, 1-24.

85. Schultz, N. A.; Dehlendorff, C.; Jensen, B. V.; Bjerregaard, J. K.; Nielsen, K. R.; Bojesen, S. E.; Calatayud, D.; Nielsen, S. E.; Yilmaz, M.; Hollander, N. H.;

Andersen, K. K.; Johansen, J. S., MicroRNA biomarkers in whole blood for detection of pancreatic cancer. Jama 2014, 311, (4), 392-404.

86. Su, Q.; Zhu, E. C.; Qu, Y. L.; Wang, D. Y.; Qu, W. W.; Zhang, C. G.; Wu, T.; Gao, Z. H., Serum level of co-expressed hub miRNAs as diagnostic and prognostic biomarkers for pancreatic ductal adenocarcinoma. Journal of Cancer 2018, 9, (21), 3991-3999.

87. Deng, T.; Yuan, Y.; Zhang, C.; Zhang, C.; Yao, W.; Wang, C.; Liu, R.; Ba, Y., Identification of Circulating MiR-25 as a Potential Biomarker for Pancreatic Cancer Diagnosis. Cellular physiology and biochemistry : international journal of experimental cellular physiology, biochemistry, and pharmacology 2016, 39, (5), 1716-1722.

88. Liu, R.; Chen, X.; Du, Y.; Yao, W.; Shen, L.; Wang, C.; Hu, Z.; Zhuang, R.; Ning, G.; Zhang, C.; Yuan, Y.; Li, Z.; Zen, K.; Ba, Y.; Zhang, C. Y., Serum microRNA expression profile as a biomarker in the diagnosis and prognosis of pancreatic cancer. Clinical chemistry 2012, 58, (3), 610-8.

89. Mayer, G., The chemical biology of aptamers. Angewandte Chemie 2009, 48, (15), 2672-89.

90. Ireson, C. R.; Kelland, L. R., Discovery and development of anticancer aptamers. Molecular cancer therapeutics 2006, 5, (12), 2957-62.

91. Djordjevic, M., SELEX experiments: new prospects, applications and data analysis in inferring regulatory pathways. Biomolecular engineering 2007, 24, (2), 179-89.

92. Kim, Y. H.; Sung, H. J.; Kim, S.; Kim, E. O.; Lee, J. W.; Moon, J. Y.; Choi, K.; Jung, J. E.; Lee, Y.; Koh, S. S.; Rhee, S. G.; Heo, K.; Kim, I. H., An RNA aptamer that specifically binds pancreatic adenocarcinoma up-regulated factor inhibits migration and growth of pancreatic cancer cells. Cancer letters 2011, 313, (1), 76-83.

93. Kim, S. A.; Lee, Y.; Jung, D. E.; Park, K. H.; Park, J. Y.; Gang, J.; Jeon, S. B.; Park, E. C.; Kim, Y. G.; Lee, B.; Liu, Q.; Zeng, W.; Yeramilli, S.; Lee, S.; Koh, S. S.; Song, S. Y., Pancreatic adenocarcinoma up-regulated factor (PAUF), a novel up-regulated 
secretory protein in pancreatic ductal adenocarcinoma. Cancer science 2009, 100, (5), 828-36.

94. Ray, P.; Rialon-Guevara, K. L.; Veras, E.; Sullenger, B. A.; White, R. R., Comparing human pancreatic cell secretomes by in vitro aptamer selection identifies cyclophilin B as a candidate pancreatic cancer biomarker. The Journal of clinical investigation 2012, 122, (5), 1734-41.

95. Ray, P.; Sullenger, B. A.; White, R. R., Further characterization of the target of a potential aptamer biomarker for pancreatic cancer: cyclophilin B and its posttranslational modifications. Nucleic acid therapeutics 2013, 23, (6), 435-42.

96. Xiong, H. Q.; Rosenberg, A.; LoBuglio, A.; Schmidt, W.; Wolff, R. A.; Deutsch, J.; Needle, M.; Abbruzzese, J. L., Cetuximab, a monoclonal antibody targeting the epidermal growth factor receptor, in combination with gemcitabine for advanced pancreatic cancer: a multicenter phase II Trial. J Clin Oncol 2004, 22, (13), 2610-6.

97. Ray, P.; Cheek, M. A.; Sharaf, M. L.; Li, N.; Ellington, A. D.; Sullenger, B. A.; Shaw, B. R.; White, R. R., Aptamer-mediated delivery of chemotherapy to pancreatic cancer cells. Nucleic acid therapeutics 2012, 22, (5), 295-305.

98. Zhang, J.; Li, S.; Liu, F.; Zhou, L.; Shao, N.; Zhao, X., SELEX aptamer used as a probe to detect circulating tumor cells in peripheral blood of pancreatic cancer patients. PloS one 2015, 10, (3), e0121920.

99. Champanhac, C.; Teng, I. T.; Cansiz, S.; Zhang, L.; Wu, X.; Zhoa, Z.; Fu, T.; Tan, W., Development of a panel of DNA Aptamers with High Affinity for Pancreatic Ductal Adenocarcinoma. Scientific reports 2015, 5, 16788.

100. Wu, X.; Zhao, Z.; Bai, H.; Fu, T.; Yang, C.; Hu, X.; Liu, Q.; Champanhac, C.; Teng, I. T.; Ye, M.; Tan, W., DNA Aptamer Selected against Pancreatic Ductal

Adenocarcinoma for in vivo Imaging and Clinical Tissue Recognition. Theranostics 2015, 5, (9), 985-94.

101. Dua, P.; S, S.; Kim, S.; Lee, D. K., ALPPL2 Aptamer-Mediated Targeted Delivery of 5-Fluoro-2'-Deoxyuridine to Pancreatic Cancer. Nucleic acid therapeutics 2015, 25, (4), 180-7.

102. Yoon, S.; Huang, K. W.; Reebye, V.; Mintz, P.; Tien, Y. W.; Lai, H. S.; Saetrom, P.; Reccia, I.; Swiderski, P.; Armstrong, B.; Jozwiak, A.; Spalding, D.; Jiao, L.; Habib, N.; Rossi, J. J., Targeted Delivery of C/EBPalpha -saRNA by Pancreatic Ductal Adenocarcinoma-specific RNA Aptamers Inhibits Tumor Growth In Vivo. Molecular therapy : the journal of the American Society of Gene Therapy 2016, 24, (6), 11061116.

103. Yoon, S.; Huang, K. W.; Reebye, V.; Spalding, D.; Przytycka, T. M.; Wang, Y.; 
Swiderski, P.; Li, L.; Armstrong, B.; Reccia, I.; Zacharoulis, D.; Dimas, K.; Kusano, T.; Shively, J.; Habib, N.; Rossi, J. J., Aptamer-Drug Conjugates of Active Metabolites of Nucleoside Analogs and Cytotoxic Agents Inhibit Pancreatic Tumor Cell Growth. Molecular therapy. Nucleic acids 2017, 6, 80-88.

104. Yoon, S.; Rossi, J. J., Treatment of Pancreatic Cancer by Aptamer Conjugated C/EBPalpha-saRNA. Advances in experimental medicine and biology 2017, 983, 173-188.

105. Kratschmer, C.; Levy, M., Targeted Delivery of Auristatin-Modified Toxins to Pancreatic Cancer Using Aptamers. Molecular therapy. Nucleic acids 2018, 10, 227236.

106. Goetze, J. P.; Nielsen, F. C.; Burcharth, F.; Rehfeld, J. F., Closing the gastrin loop in pancreatic carcinoma: coexpression of gastrin and its receptor in solid human pancreatic adenocarcinoma. Cancer 2000, 88, (11), 2487-94.

107. Clawson, G. A.; Abraham, T.; Pan, W.; Tang, X.; Linton, S. S.; McGovern, C. O.; Loc, W. S.; Smith, J. P.; Butler, P. J.; Kester, M.; Adair, J. H.; Matters, G. L., A Cholecystokinin B Receptor-Specific DNAAptamer for Targeting Pancreatic Ductal Adenocarcinoma. Nucleic acid therapeutics 2017, 27, (1), 23-35.

108. Kim, Y. J.; Lee, H. S.; Jung, D. E.; Kim, J. M.; Song, S. Y., The DNA aptamer binds stemness-enriched cancer cells in pancreatic cancer. Journal of molecular recognition : JMR 2017, 30, (4).

109. Park, J. Y.; Cho, Y. L.; Chae, J. R.; Moon, S. H.; Cho, W. G.; Choi, Y. J.; Lee, S. J.; Kang, W. J., Gemcitabine-Incorporated G-Quadruplex Aptamer for Targeted Drug Delivery into Pancreas Cancer. Molecular therapy. Nucleic acids 2018, 12, 543-553.

110. Ryan, D. P.; Hong, T. S.; Bardeesy, N., Pancreatic adenocarcinoma. The New England journal of medicine 2014, 371, (11), 1039-49.

111. Collins, M. A.; Pasca di Magliano, M., Kras as a key oncogene and therapeutic target in pancreatic cancer. Frontiers in physiology 2013, 4, 407.

112. Bryant, K. L.; Mancias, J. D.; Kimmelman, A. C.; Der, C. J., KRAS: feeding pancreatic cancer proliferation. Trends in biochemical sciences 2014, 39, (2), 91-100.

113. Cogoi, S.; Zorzet, S.; Rapozzi, V.; Geci, I.; Pedersen, E. B.; Xodo, L. E., MAZ-binding G4-decoy with locked nucleic acid and twisted intercalating nucleic acid modifications suppresses KRAS in pancreatic cancer cells and delays tumor growth in mice. Nucleic acids research 2013, 41, (7), 4049-64.

114. Cogoi, S.; Jakobsen, U.; Pedersen, E. B.; Vogel, S.; Xodo, L. E., Lipid-modified G4decoy oligonucleotide anchored to nanoparticles: delivery and bioactivity in pancreatic cancer cells. Scientific reports $2016,6,38468$. 
115. Liau, S. S.; Whang, E., HMGA1 is a molecular determinant of chemoresistance to gemcitabine in pancreatic adenocarcinoma. Clin Cancer Res 2008, 14, (5), 1470-7.

116. Hassan, F.; Ni, S.; Arnett, T. C.; McKell, M. C.; Kennedy, M. A., AdenovirusMediated Delivery of Decoy Hyper Binding Sites Targeting Oncogenic HMGA1

Reduces Pancreatic and Liver Cancer Cell Viability. Molecular therapy oncolytics 2018, 8, 52-61. 
Peer-reviewed version available at Int. J. Mol. Sci. 2019, 20, 3331; doi:10.3390/iims20133331

Table 1. Clinical trials of Oligonucleotide therapeutics for patients with pancreatic cancer

\begin{tabular}{ccccccc}
\hline Trial identifer & Targeted drug & \multicolumn{2}{c}{ Target molecule Category of agent } & Enrollment & Organizing location & Study phase \\
\hline NCT00005594 & ISIS 2503 & Hras & Antisense & 48 & United States & Phase 2 \\
NCT00844064 & AP 12009 / OT-101 & TGF- $\beta 2$ & Antisense & 61 & Germany & Phase $1 / 2$ \\
NCT02983578 & AZD9150 & STAT3 & Antisense & 75 (estimated) & United States & Phase 2 \\
NCT00557596 & AEG35156 & XIAP & Antisense & 14 & United States & Phase 1 \\
NCT01844817 & OGX-427 / apatorsen & Hsp27 & Antisense & 132 & United States & Phase 2 \\
NCT01808638 & Atu027 & PKN3 & siRNA & 29 & Germany & Phase 1/2 \\
NCT01188785 & siG12D LODER & KrasG12D & siRNA & 15 & United States & Phase 1 \\
NCT01676259 & siG12D LODER & KrasG12D & siRNA & 80 (estimated) & United States & Phase 2 \\
NCT03608631 & iExosomes & KrasG12D & siRNA & 28 (estimated) & United States & Phase 1 \\
NCT03432624 & Detection Kit & MiR-25 & miRNA & 750 (estimated) & China & \\
\hline TGF- $\beta 2:$ transforming growth factor-beta 2; XIAP: X-Linked Inhibitor of Apoptosis; PKN3: protein kinase N3; Hsp27: heat shock protein 27
\end{tabular}

Table 2. The utilizing methods of aptamer for pancreatic cancer

\begin{tabular}{cccc}
\hline No. & Target molecule & How to work? & Ref. \\
\hline 1 & P12FR2 & Oncosuppressor & {$[92]$} \\
2 & C/EBPa-saRNA & Oncosuppressor & {$[104]$} \\
3 & cyclophilin B & Biomarker & {$[94,95]$} \\
4 & circulating tumor cells & Biomarker & {$[98]$} \\
5 & EGFR & Targeted delivery & {$[97]$} \\
6 & ALPPL2 & Targeted delivery & {$[101]$} \\
7 & ApDCs P19 & Targeted delivery & {$[103]$} \\
8 & Auristatin-Modified Toxins & Targeted delivery & {$[105]$} \\
9 & CCKBR & Targeted delivery & {$[107]$} \\
10 & APTA-12 & Targeted delivery & {$[109]$} \\
11 & XQ-2d & Detector & {$[100]$} \\
12 & cancer stem cells & Detector & {$[108]$} \\
\hline
\end{tabular}

P12FR2: 2-fluoropyrimidine modified RNA aptamer

SELEX: systematic evolution of ligands by exponential enrichment

C/EBPa: CCAAT/enhancer-binding protein- $\alpha$

EGFR: epidermal growth factor receptor

ALPPL2: alkaline phosphatase placental-like 2

ApDCs: Aptamer-drug conjugates

CCKBR: cholecystokinin $B$ receptor

APTA-12: a gemcitabine-incorporated AS1411

XQ-2d: a truncated DNA aptamer 


\section{Figuer legends}

Figure 1. Schematic of oligonucleotide activities in disease progression

Decoys bind to transcription factors of targeted DNA at the earliest phase.

Subsequently, antisense RNA, siRNA and miRNA act to target mRNAs.

Then, aptamers directly inhibit proteins in the process of disease development.

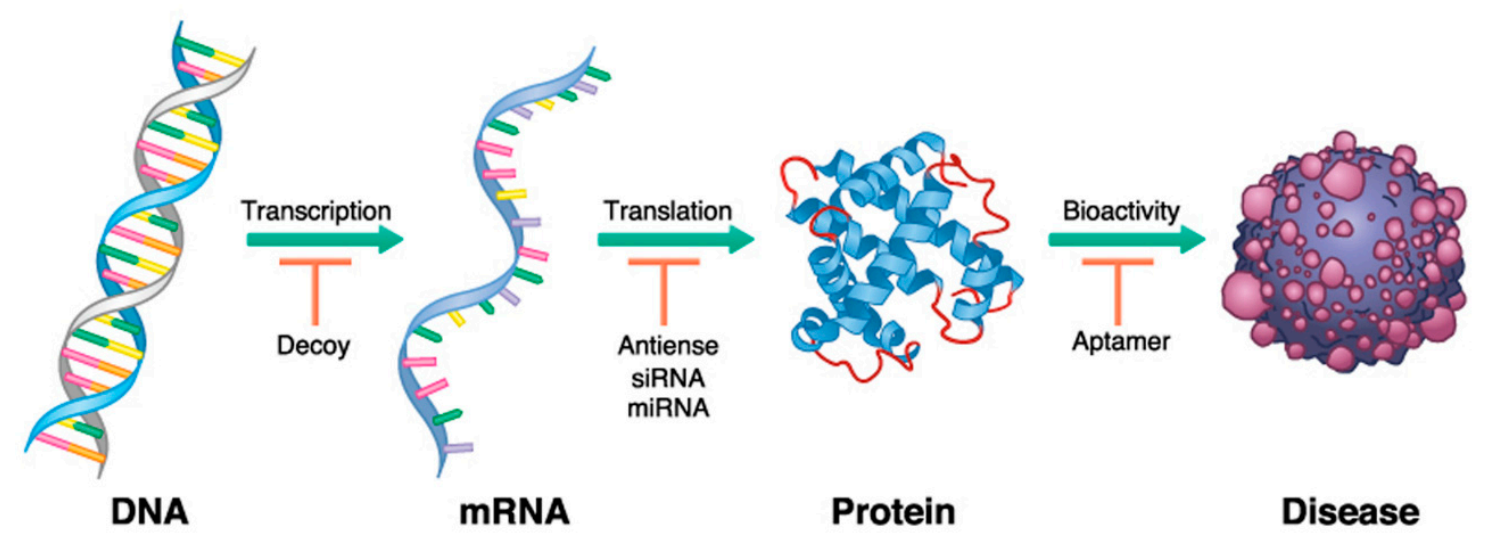




\section{Figure 2. STNM01 blocks the glycosaminoglycan synthesis pathway}

CS-E is a matrix glycosaminoglycan (GAG), a linear polysaccharide composed of a repeating disaccharide unit containing D-glucuronic acid (GlcA) and N-acetyl-Dgalactosamine (GalNAc), whose 4 and 6 positions are sulfated. CHST15 is a type 2 transmembrane Golgi protein that transfers sulfate to position 6 of GalNAc (4SO4) residues of CS-A to yield CS-E.

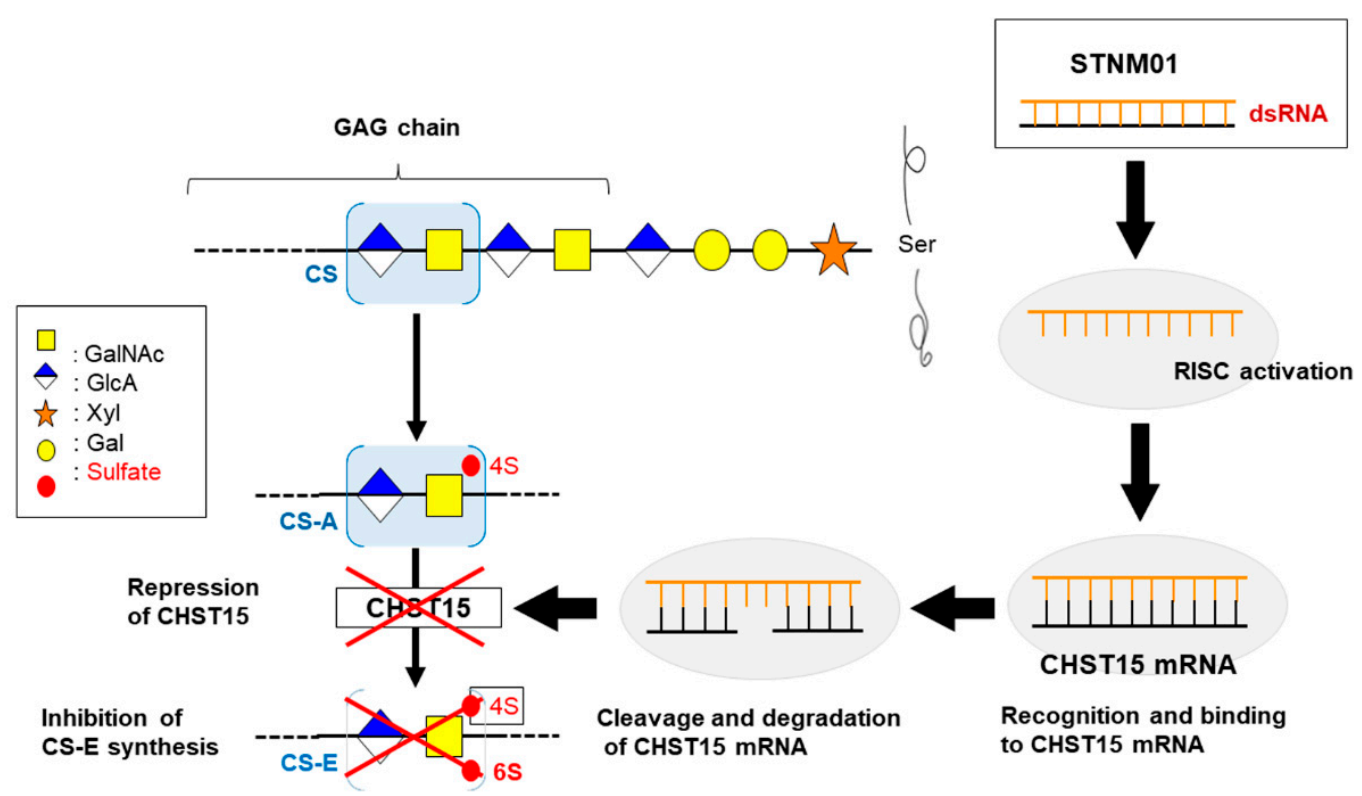

